\title{
Gyroresonance in unsaturated composite bodies: Experiments and theory
}

\author{
Jean-Luc Mattei, David Bariou, Alexis Chevalier, and Marcel Le Floc'h \\ Laboratoire d'Electronique et des Systèmes de Télécommunications, UMR CNRS 6616, \\ Université de Bretagne Occidentale, Brest, France
}

\begin{abstract}
The permeability spectra of composite magnetic materials (NiZn ferrite composites and YIG composites) has been experimentally studied on a wide range in magnetic load $C$. The resonance frequency decreases with the increasing magnetic load of the mixture. This is attributed to the fading of the magnetic pole on the surface of the magnetic inclusions. A theory is developed which is intended to give an expression for the gyroresonance frequency of magnetic composites in their unsaturated magnetic state. It fits the experimental data over its full range of volume fraction $C$ without any corrective factors. The proposed relation, which is an extension of the Kittel's expression for the resonance frequency of a saturated body, points out a noteworthy close link between the concept of reciprocity and the one of demagnetization. (C) 2000 American Institute of Physics. [S0021-8979(00)53508-7]
\end{abstract}

\section{INTRODUCTION}

In order to make satisfactory predictions about the behavior of a material intended to be manufactured, it is useful to benefit from laws describing its relevant properties in terms of meaningful physical parameters. Among the laws widely exploited in designing materials for microwave applications, the Snoek's law ${ }^{1}$ and the Kittel's relation ${ }^{2}$ can be mentioned as they are certainly the most currently used for their good reliability when applied to bulk materials. In counterpart there is a lack of any simple relation which could confidently predict the behavior of magnetic composite materials.

This paper presents a straight relation, which describes the particular behavior exhibited by the resonance frequency, occurring in the microwave range, of unsaturated magnetic composite materials. It points out a close relation which exists between, on one hand, the demagnetizing factor attributed to a magnetic inclusion of the composite, and on the other hand, a reciprocity principle derived to be applied to composite mixtures.

\section{EXPERIMENTS: PROCEDURE AND RESULTS}

Powders of YIG and $\mathrm{Ni}-\mathrm{Zn}$ ferrites were prepared by mechanical grinding of sintered bulk materials. Particles were sized to $10 \mu \mathrm{m}$ for the YIG and about $0.1 \mu \mathrm{m}$ for the $\mathrm{Ni}-\mathrm{Zn}$ ferrites. In both cases particles have polydomain structures with isotropic properties well described by a scalar magnetic susceptibility. Composite materials were produced by mixing nonmagnetic powder (resin) with soft magnetic powder, then by pressing the mixtures in ring-shaped molds at room temperature. The prepared composite samples have a volume loading $C$ in magnetic matter contained between $5.1 \%$ and $69.5 \%$. The complex susceptibility $\left(\chi^{\prime}-j \chi^{\prime \prime}\right)$ has been investigated at room temperature in the $30 \mathrm{MHz}-20$ $\mathrm{GHz}$ frequency band using network analyzers (HP 8753 and HP 8720) and a coaxial line filled with the toroidal samples in the APC7 standard. The magnetization mechanism concerning the powder materials this work deals with, and in spite of their initial polydomain character, is controlled primarily by spin rotations in the explored frequency range (see the inset in Fig. 1, and also Ref. 3).

For several volumic fractions $C$, the frequency $f_{R}$ to which $\chi^{\prime \prime}$ reaches its maximum value is reported. As shown in Fig. $1, f_{R}$ decreases with the increase in volume loading of magnetic matter. Previous works ${ }^{4-6}$ report on some experimental results in favor of this kind of behavior of $f_{R}$. The next sections discuss to what extent this behavior can be attributed to the demagnetizing field generated by the magnetic poles on the surface of the magnetic inclusions.

\section{THE RECIPROCITY FACTOR}

Considering two magnetization distributions $\left(\mathbf{M}_{1}\right.$ and $\mathbf{M}_{2}$ ) occupying two regions of volume $V_{i}(i=1$ or 2$)$, the magnetic flux density $\mathbf{B}_{2}$ produced by $\mathbf{M}_{1}$ throughout the

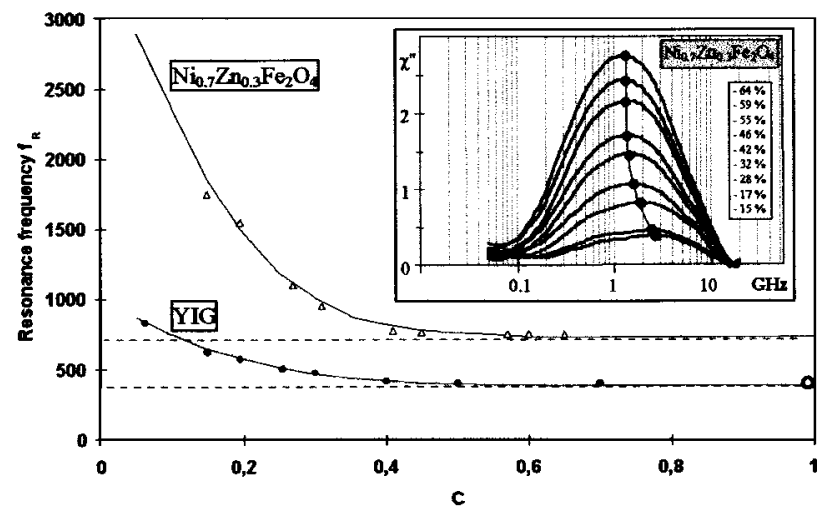

FIG. 1. Gyroresonance in unsaturated magnetic composites vs the volumic fraction in magnetic matter $C$. The symbols are the experimental data, the full lines are the fits provided by Eq. (8) with the following values for the YIG: $M_{S}=140 \mathrm{kA} / \mathrm{m}, H_{0}=11.4 \mathrm{kA} / \mathrm{m}, \quad \alpha=0.3, \chi_{i}=21$; and for the $\mathrm{Ni}_{0.7} \mathrm{Zn}_{0.3} \mathrm{Fe}_{2} \mathrm{O}_{4}: M_{S}=410 \mathrm{kA} / \mathrm{m}, H_{0}=21.3 \mathrm{kA} / \mathrm{m}, \alpha=0.3, \chi_{i}=11$. It may be said that magnetic composites with $C$ higher than around 0.25 have the same resonance frequency as the bulk itself (dashed lines).The open circle is the theoretical value for the bulk YIG [A. Globus, J. Phys C1 38, 1 (1977)]. The spectra of dynamic losses shown in the inset gives a strong argument in favor of magnetization mechanism only controlled by spin rotations $(0.15$ $<C<0.64)$. 
volume $V_{2}$ is linked to the magnetic flux density $\mathbf{B}_{1}$ produced by $\mathbf{M}_{2}$ throughout the volume $V_{1}$ by the reciprocity theorem, proved by Brown, as follows ${ }^{7}$

$$
\int_{V_{2}} \mathbf{B}_{1} \cdot \mathbf{M}_{2} d \tau=\int_{V_{1}} \mathbf{B}_{2} \cdot \mathbf{M}_{1} d \tau .
$$

However, as it will be shown in the following, if the principle of reciprocity is intended to be applied to unsaturated composite mixtures, it must be expressed as an extended form of Eq. (1). The composite mixtures considered in this work are constituted of magnetic (volumic fraction $C$ ) and nonmagnetic (volumic fraction 1-C) particles dispersed at random. It will be assumed the inclusions are aggregated in clusters, the sizes of which are supposed to depend on $C$ only. Effective medium theories (EMTs) describe composite materials in term of effective permeability, which characterizes an equivalent medium. ${ }^{8}$ Among the available EMTs providing simple, analytical, and nonempirical expressions for the effective permeability, the Bruggeman self-consistent theory ${ }^{8}$ is known to predict the existence of a percolation threshold $C_{P}\left(C_{P}=1 / 3\right.$ in the basic form of this EMT $)$.

In the dipolar approximation, on which this EMT is based, the uniform magnetic flux density inside the volume of a spherical particle (permeability $\mu_{i}$, uniform magnetization $\mathbf{M}_{i}$ )immersed in the homogeneous equivalent medium (permeability $\mu_{e}$, uniform magnetization $\mathbf{M}_{e}$ ) with uniform magnetic field $\mathbf{H}_{0}$ is $\mathbf{B}_{i}=\mu_{i} \mathbf{H}_{i}$, and its magnetization is $\mathbf{M}_{i}$ $=\chi_{i} \mathbf{H}_{i}$, where ${ }^{9}$

$$
\mathbf{H}_{i}=3 \frac{\mu_{e}}{2 \mu_{e}+\mu_{i}} \mathbf{H}_{0},
$$

therefore, one gets

$$
\begin{aligned}
& \int_{V} \mathbf{B}_{i} \mathbf{M}_{e} d \tau=\frac{3 \mu_{i} \mu_{e}}{2 \mu_{e}+\mu_{i}} \int_{V} \mathbf{H}_{e} \mathbf{M}_{e} d \tau, \\
& \int_{V} \mathbf{B}_{e} \mathbf{M}_{i} d \tau=\frac{3 \chi_{i} \mu_{e}^{2}}{2 \mu_{e}+\mu_{i}} \frac{1}{\chi_{e}} \int_{V} \mathbf{H}_{e} \cdot \mathbf{M}_{e} d \tau,
\end{aligned}
$$

where the integrations are over the composite's volume. Taking the member to member ratio of Eqs. (3) leads to the following noteworthy relations:

$$
\int_{V_{i}} \mathbf{B}_{i} \mathbf{M}_{e} d \tau=R \int_{V_{e}} \mathbf{B}_{e} \mathbf{M}_{i} d \tau,
$$

with

$$
R=\frac{\mu_{i} \chi_{e}}{\mu_{e} \chi_{i}}
$$

(The integrations have been reduced to the volumes where the magnetizations are nonzero.)

It appears that relations (1) and (4a) are strictly the same in two particular cases only: either if both materials (i.e., the magnetic particle and its surrounding equivalent medium) are saturated $\left[\mu_{\mathrm{i}}=\mu_{\mathrm{e}}=1\right.$, the situation to which Eq. (1) refers] or if they are identical $\left(\mu_{\mathrm{i}}=\mu_{\mathrm{e}}\right)$. But in the general case of unsaturated material, with which this paper deals, $R$ belongs to the range $[0,1]$. Therefore, Eq. (4) predicts the way in which the reciprocity is verified. It depends on the value of the ratio $R$, which will be called reciprocity factor.

It can be attempted to link the concept of reciprocity to the one of demagnetization. Actually the state of demagnetization of a body depends on its shape through the continuity of the normal magnetic flux density component on its external surface, whereas the reciprocity principle deals with the flux density throughout the volume of this body. Let us consider the simple case of a magnetic ellipsoid characterized by its permeability $\mu_{i}$ and its shape factor ${ }^{9} \mathbf{A}$ (expressed as a diagonal tensor of components $A_{\alpha}$ in the particle frame, $\alpha$ $=x, y$, or $z$ ) immersed in the continuous medium of permeability $\mu_{e}$. The extent to which the external field $\mathbf{H}_{0}$ is decreased by the magnetization $\mathbf{M}_{i}$ is given by the demagnetizing tensor $\mathbf{N}$ in the following expression: $\mathbf{H}_{i}=\mathbf{H}_{0}$ $-\mathbf{N M}_{i}$. From this definition of the demagnetizing factor $\mathbf{N}$, it comes out that its diagonal components $N_{\alpha}$ are $^{9}$

$$
N_{\alpha}=\frac{\mu_{i}-\mu_{e}}{\mu_{e}\left(\mu_{i}-1\right)} A_{\alpha} .
$$

The reliability of this equation has been successfully verified by using numerical simulations. ${ }^{10}$ It is clear from Eq. (5) that the demagnetization factor $\mathbf{N}$ depends on the shape of the magnetic inclusion, as well as of its surrounding medium. Indeed when the host is the empty space $\left(\mu_{e}=1\right)$, one gets $N_{\alpha}=A_{\alpha}$ (noted $N_{\alpha}^{0}$ below), whereas $0<N_{\alpha}<A_{\alpha}$ when $\mu_{e}$ $>1$. The demagnetizing field $\mathbf{H}=-\mathbf{N} \mathbf{M}_{i}$ is generated by the magnetic poles on the inclusion surface. Therefore, Eq. (5) can be understood as an illustration of the magnetic poles fading which is intuitively expected to arise for a magnetic body included in a composite medium, when the magnetic load is increased. Keeping this in mind, we propose to express $N_{\alpha}$ in the following attractive form, which reflects clearly the magnetic poles fading described above:

$$
N_{\alpha}=A_{\alpha}(1-R)
$$

where $R$ is the reciprocity factor given by Eq. (4b).

The influence of $C$ on the reciprocity factor $R$, and therefore the variation of the demagnetization factor coefficients $N_{\alpha}$, are obtained through the expression for the effective permeability $\mu_{e}$. Going back to the Bruggeman's EMT, the self-consistent relation $\left\langle\mu_{i} \mathbf{H}_{i}\right\rangle=\mu_{e}\left\langle H_{e}\right\rangle$, where \langle\rangle denotes the average over the various types of inclusions, leads to the desired expression ${ }^{11}$ of $\mu_{e}$.

The variations of $R$ [Eq. (4b)] and of $N_{\alpha}$ [Eq. (5)] as functions of $C$ are shown in Fig. 2, in the case of spherical inclusions $\left(A_{\alpha}=1 / 3\right)$. We think that both are correlated to a progressive decrease of the magnetic pole density. Their typical trend is to tend to a constant (the value of which is characteristic of a bulky homogeneous material) when the percolation threshold $\left(C_{P}=1 / 3\right)$ is reached. This behavior might show that any two particles in the composites can be considered as interconnected by a magnetic path only if $C$ is higher than the percolation threshold. The role of percolation is more pronounced if high permeability $\left(\mu_{i}\right)$ materials are used. 


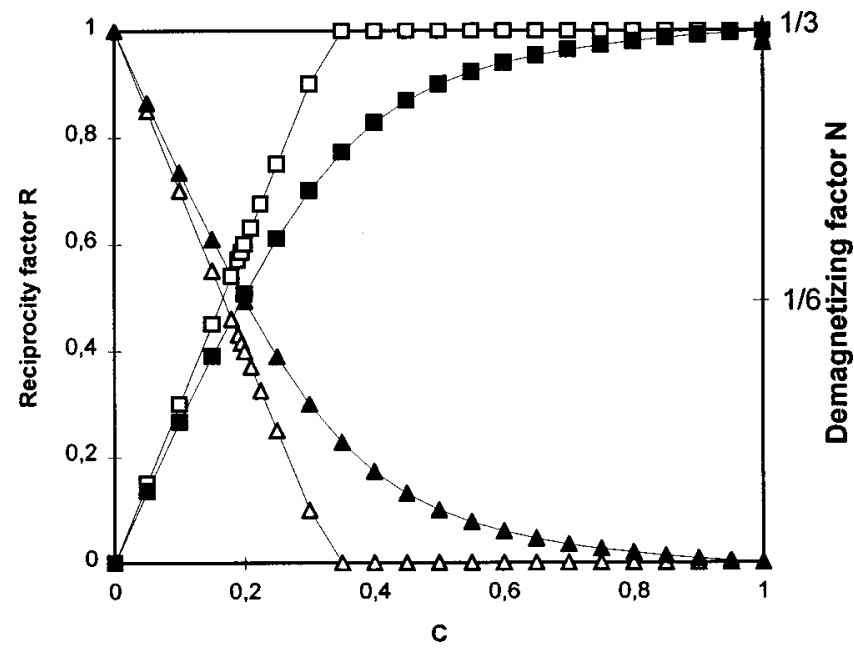

FIG. 2. Demagnetizing factor [Eq. (6), triangles] of a spherical particle included in a composite material, and reciprocity factor [Eq. (4b), squares] for the composite material, vs the volumic fraction in magnetic matter. The magnetic permeability of the particle has been taken equal to 20 (full symbols) or to infinity (open symbols).

\section{GYRORESONANCE IN COMPOSITE BODIES}

In order to test experimentally the role played by the reciprocity factor in the demagnetization of unsaturated magnetic composites, this section proposes an interpretation of the gyroresonance measured on soft composite materials.

Actually, as first shown by Kittel, ${ }^{2}$ the condition for the gyroresonance of a body depends strongly on its shape. The Kittel equation for the resonance frequency, which deals with an ellipsoid in the saturated magnetic state (with the magnetization $\mathbf{M}_{S}$ ), placed in the empty space and characterized by the demagnetization factors $N_{\alpha}^{0}$ (in fact, $N_{\alpha}^{0}=A_{\alpha}$ in this situation ${ }^{9}$ ) is

$$
f_{K}=\sqrt{f_{0}+f_{M}\left(N_{y}^{0}-N_{z}^{0}\right)} \times\left(1+\alpha^{2}\right)^{-1 / 2} .
$$

In this relation $f_{0}=\gamma H_{0} / 2 \pi$ (with $\gamma=35 \times 12^{10^{-3}} \mathrm{MHz} /$ $\mathrm{Am}^{-1}$, and where $\mathbf{H}_{0}$ is the crystalline anisotropy field responsible for the magnetic moments alignment, its direction defines the $z$ axis. No external static field is considered), $f_{M}=\gamma M_{S} / 2 \pi$ and $\alpha$ is the damping coefficient. ${ }^{12}$

According to the EMT, the description of the composite material consists of an ellipsoidal particle included in the effective medium. In order to make Eq. (7) suitable to be applied to such a situation, in the expression of $f_{K}$ it is suggested to substitute the demagnetizing factor $N_{\alpha}^{0}$ by $N_{\alpha}$, as given by Eq. (6). Therefore, the proposed extension of the Kittel expression (7), initially written for a saturated ellipsoid, to the frequency resonance $f_{R}$ of an unsaturated mixture formed by the assembly of saturated ellipsoids randomly dispersed in a nonmagnetic matrix, is written as

$$
f_{R}=\left[f_{0}+f_{M} \times\left(N_{y}^{0}-N_{z}^{0}\right) \times(1-R)\right] \times\left(1+\alpha^{2}\right)^{-1 / 2},
$$

which points out the important role taken by the reciprocity factor $R$. Figure 2 shows how Eq. (8) matches very satisfac- torily our experimental data (The magnetic parameters that have been used are given in the caption of Fig. 1.) In particular, a quite good approximation would be to write $f_{R}$ $=f_{0}$ from a magnetic load $C=0.25$ up to $C=1$. To perform these fits, one has to know accurately the values taken by the factor $A_{\alpha}$, which characterizes the inclusions shape at a given volumic fraction $C$. As $C$ increases the individual particles stick together to form complex chains and clusters. Hence the form factor $A_{\alpha}$ to be used in the EMT must reflect this variety of shapes, consequently these $A_{\alpha}$ 's are lower than $1 / 3$. Their values are independent of the average isotropy of the material because the EMT is based on an average of the fields, not of the $A_{\alpha}$ 's (which appear in the denominator of the fields). Then the experimental values for the shape factor are obtained by inverting the self-consistent equation $\left\langle\mu_{i} \mathbf{H}_{i}\right\rangle=\mu_{e}\left\langle H_{e}\right\rangle$ in the static limit. ${ }^{13}$ The value of $A_{\alpha}$ is about 0.25 if $C$ is near $5 \% .{ }^{13}$ In the range $5 \%-20 \%$, the shape factor increases until it reaches the value of $1 / 3$. The isotropic shape of the aggregates is recognized from this characteristic value. About the value found for the damping parameter $(\alpha=0.3)$, note that previous determinations of $\alpha$ obtained from the Landau-Lifschitz equation ${ }^{14}$ also strongly differ from the results obtained in ferromagnetic resonance experiments (where $\alpha \sim 10^{-3}-10^{-2}$ ).

It must be underlined that the value of $C=20 \%$ to which the shape factor reaches its maximum value of $1 / 3$ ) is numerically equal, or nearly so, to the site percolation threshold $\left(C_{P}=18 \%\right)$ given for a three-dimensional structure of equalsized randomly packed hard spheres. ${ }^{15,16}$ It can be easily verified that the variation of $f_{R}$ around $C_{P}$ is as sharp as the permeability $\mu_{i}$ is high. When the permeability $\mu_{i}$ takes an infinite value, $f_{R}$ reaches the bulk resonance frequency $f_{0}$ for a volume concentration $C \approx 20 \%$.

${ }^{1}$ J. L. Snoek, Physica (Amsterdam) 14, 207 (1948).

${ }^{2}$ C. Kittel, Phys. Rev. 73, 155 (1948).

${ }^{3}$ G. T. Rado, R. W. Wright, and W. H. Emerson, Phys. Rev. 80, 273 (1950).

${ }^{4}$ A. Berthault, D. Rousselle, and P. G. Zerah, J. Magn. Magn. Mater. 112, 477 (1992).

${ }^{5}$ D. Rousselle, A. Berthault, O. Acher, J. P. Bouchaud, and P. G. Zerah, J. Appl. Phys. 74, 475 (1993).

${ }^{6}$ T. Tsutaoka, M. Ueshima, T. Tokunaga, T. Nakamura, and K. Hatakeyama, J. Appl. Phys. 78, 3983 (1995).

${ }^{7}$ W. F. Brown, Magnetostatic Principles in Ferromagnetism (NorthHolland, Amsterdam, 1962).

${ }^{8}$ R. Landauer, Electrical Transport and Optical Properties of Inhomogeneous Media, edited by J. C. Garland and D. B. Tanner (AIP, New York, 1978).

${ }^{9}$ J. A. Stratton, Electromagnetic Theory (McGraw-Hill, New York, 1941).

${ }^{10}$ J. L. Mattei and M. Le Floc'h, J. Magn. Magn. Mater. (to be published).

${ }^{11}$ D. Rousselle, A. Berthault, O. Acher, J. P. Bouchaud, and G. Zérah, J. Appl. Phys. 74, 475 (1993).

${ }^{12}$ T. L. Gilbert, Phys. Rev. 100, 1243 (1955).

${ }^{13}$ J. L. Mattei, P. Laurent, A. Chevalier, and M. Le Floc'h, J. Phys. IV, C1-547 (1997).

${ }^{14}$ J. P. Bouchaud and P. G. Zerah, J. Appl. Phys. 67, 5512 (1990)

${ }^{15}$ M. J. Powell, Phys. Rev. B 20, 4194 (1979).

${ }^{16}$ D. Stauffer and A. Aharoni, Introduction to Percolation Theory (Taylor and Francis, 1992). 